\title{
Pituitary
}

\author{
Mohamad Maghnie ${ }^{a}$, Andrea Secco ${ }^{b}$ and Sandro Loche \\ aDepartment of Pediatrics, Istituto di Ricovero e Cura a Carattere Scientifico, Giannina Gaslini, University of \\ Genova, Italy \\ bepartment of Pediatrics, University of Pavia, Italy \\ 'Ospedale Regionale per le Microcitemie, Cagliari, Italy
}

\begin{abstract}
Among the wide range of relevant subjects assessed, we found that the most deserving topics in the pituitary field during the timeframe represented were: gene research, clinical trials, pathogenic mechanisms and clinical practice recommendations. We hope that this chapter will serve as a guide to orient endocrine specialists in these exciting and challenging new directions. It is undeniable that these areas have been changing so rapidly in recent years that a practicing physician inevitably finds it difficult to keep up with all of the latest developments. Therefore, a compact review and summary like the present chapter hopes to satisfy updating needs while helping busy physicians save time as well.
\end{abstract}

\section{Mechanism of the year}

\section{A comprehensive description of the transcriptome of the hypothalamoneurohypophyseal system in euhydrated and dehydrated rats}

Hindmarch C, Yao S, Beighton G, Paton J, Murphy D

The Henry Wellcome Laboratories for Integrative Neuroscience and Endocrinology, University of Bristol, Bristol, UK Proc Natl Acad Sci USA 2006;103:1609-1614

Background: The endocrine hypothalamoneurohypophyseal system (HNS) is crucially involved in water homeostasis through three interdependent mechanisms including thirst, vasopressin (AVP) and the kidneys. Small changes in plasma osmolality lead to AVP secretion promoting water conservation in its major target organ. The functional plasticity of the NHS during dehydration in terms of the gene expression involved in this pathway is not yet clearly understood.

Methods: Adult male rats were dehydrated and subsequently tissue collection from the brain and RNA extraction were carried out. The GeneSpring catalog of a list of genes identified as being expressed in the rat NHS which includes large peptidergic magnocellular neurons of the supraoptic (SON) and paraventricular hypothalamic nuclei (PVN) or neurointermediate lobe (NIL) was used. The corresponding gene lists represent comprehensive descriptions of the RNA populations expressed in the different tissue components of the HNS. Combined lists were filtered to identify genes that are putatively upregulated by at least 2-fold after dehydration or putatively downregulated by at least 2-fold after dehydration. Merging of the statistically filtered up- or downregulated gene lists gave the catalogs of transcripts that are abundantly changed in the HNS structures.

Results: Marked changes in the HSN steady-state RNA level after dehydration were evident in the SON, PVN and NIL. There were clear transcriptome phenotypic differences between the SON and PVN in the controls compared to the dehydrated tissues.

Conclusion: A large number of genes were identified as being expressed in rat HNS tissues after dehydration. The pattern of HNS transcripts with marked differences in gene expression indicates that these genes are candidate regulators and effectors of HNS activity and remodeling.

As systems biology gets into problems related to our field, we have to start looking at genomic arrays rather than single genes, at proteomics rather than single proteins and at metabolomics rather than single metabolites. Microarray analysis is an approach for expression profiling that provides the means to perform parallel analysis of tens of thousands of genes in a single assay. The results offer a quantitative assessment of whether a gene is expressed in a specific tissue or organ and whether specific transcripts are up- or downregulated or remain unchanged as a consequence of physiological and 
pathological cues. This study produced a comprehensive catalog of the genes expressed in the HNS and described how gene expression changes in the different areas of this remarkable model system in response to dehydration. There were, in fact, morphological and functional differences in the molecular phenotype of the SON and PVN, while the potential implications remain to be assessed. Various endocrine disorders or experimental conditions seem suitable for the application of this highly sophisticated method. These data may well form the basis of many future physiological studies on the gene and cell networks regulating endocrine hypothalamoneurohypophyseal function. These gene lists are freely available for download, and the raw data are in the Gene Expression Omnibus database at the National Center for Biotechnology Information (www.ncbi.nlm.nih.gov/geo). Now that you understand system biology, take a look at biological networks.

\section{New paradigms}

\section{Revealing the large-scale network organization of growth hormone-secreting cells}

Bonnefont X, Lacampagne A, Sanchez-Hormigo A, Fino E, Creff A, Mathieu MN, Smallwood S, Carmignac D,

Fontanaud P, Travo P, Alonso G, Courtois-Coutry N, Pincus SM, Robinson IC, Mollard P

Department of Endocrinology, Centre National de la Recherche Scientifique, Institut National de la Sante et de la

Recherche Médicale U661, Universities of Montpellier 1 and 2, France

Proc Natl Acad Sci USA 2005;102:16880-16885

Background: GH is secreted from the pituitary gland in an episodic manner. The GH-secreting cells are the major population of endocrine cells in the pituitary. To secrete highly ordered GH pulses, the GHsecreting cells need to mount coordinated responses to GH secretagogues. The authors have analyzed both positioning and signaling of $\mathrm{GH}$ cells in 3D histological studies.

Methods: Positioning and signaling of GH cells were analyzed using reconstructive, two-photon excitation microscopy to image the entire pituitary gland of GH-EGFP transgenic mice. In fixed pituitaries, fluorescence images were acquired in $x-y$ mode with a Zeiss LSM NLO confocal system. Mouse GH and EGFP in either the pituitary or cell incubation medium were assayed by RIA. Cytosolic calcium was also monitored.

Results: The study unveiled a homologous continuum of GH cells connected by adherens junctions that wired the whole gland and exhibited: robustness of architecture across lifespan; modularity correlated with pituitary GH contents and body growth, and connectivity with spatially stereotyped motifs of cellsynchronization-coordinating cell activity.

Conclusion: The study describes an approach for studying the network that assembles a population of homogeneous cell types, as well as for exploring how this system is maintained through normal cell turnover and replacement.

Growth hormone secretion is regulated by the coordinated action of growth hormone-releasing hormone (GHRH) and somatostatin (SRIH), which respectively stimulate and inhibit its release from the pituitary gland. The hypothalamic release of GHRH and of SRIH are, in turn, subject to regulation by a complex network of brain neurotransmitters and neuropeptides. GH secretion is also influenced by metabolic and hormonal signals from the periphery (including glucocorticoids, thyroid hormones and sex steroids) which may modulate somatotroph function, either directly or via the hypothalamus. In addition, GH regulates its own secretion by a feedback mechanism functioning either through direct action or through effects mediated by the IGFs and possibly by other metabolic factors such as FFA insulin and glucose. The result of this complex regulation is an episodic pattern of secretion throughout the 24-hour cycle with an amplification of GH peaks during the first hours of sleep. The functional organization of GH cells that might explain the coordinated responses of the numerous individual cells is still poorly understood. The paper shed lights on this organization by showing that $\mathrm{GH}$-secreting cells are in fact a geometrically connected homotypic network of cells whose local morphology and connection can vary in such a way as to alter the timing of cellular responses in order to promote more coordinated pulsatile secretion. Developmental or pathological disruption of this 
architecture may explain some gradually-developing $\mathrm{GH}$-deficient states such as those following traumatic brain injury or cranial irradiation and could well contribute to the uncoordinated GH profiles seen in subjects previously described as exhibiting 'neurosecretory dysfunction'. One can imagine that the development of non-invasive imaging of the present 3D network might be applied in diagnosis of some gradually developing GH-deficiency states.

\section{Concepts revised}

\section{HESX1 expression in human normal pituitaries and pituitary adenomas}

Mantovani G, Asteria C, Pellegrini C, Bosari S, Alberti L, Bondioni S, Peverelli E, Spada A, Beck-Peccoz P Institute of Endocrine Sciences, Ospedale Maggiore IRCCS, University of Milan, Milan, Italy Mol Cell Endocrinol 2006:247:135-139

Background: Hesx1 is a member of the paired-like class of homeobox genes and is one of the earliest markers expressed in the anterior midline visceral endoderm with subsequent resolution of expression to the ventral diencephalon and Rathke's pouch. Expression continues in the developing anterior pituitary until embryonic day E12.0, when its transcripts disappear in a spatiotemporal sequence that corresponds to progressive pituitary cell differentiation, expression being undetectable by E13.5. The authors investigated whether Hesx1 is expressed during adult life in human normal pituitaries and in different types of human pituitary adenomas.

Methods: Tissues from post-mortem human normal pituitaries and from 19 patients with non-secreting and secreting pituitary adenomas were obtained. The expression and quantification of HESX1 mRNAs was examined by using the quantitative RT-PCR method.

Results: HESX1 expression was found in normal adult pituitaries as well as in pituitary tumor tissues. $\mathrm{GH}$-secreting adenomas, non-secreting adenomas, ACTH-secreting adenomas, PRL-secreting adenomas and TSH-secreting-adenomas displayed similar expression. Western blot analysis confirmed the presence of the HESX1 protein in both the normal pituitary and pituitary adenomas.

Conclusion: HESX1 mRNA and protein were detected in normal pituitary and in pituitary adenomas regardless of hormone phenotype, tumor size or invasiveness. These data suggest that HESX1 expression continues during adult life.

Hesx 1 is at the center of a gene network required for proper differentiation of the anterior pituitary, as well as of other parts of the central nervous system. Hesx 1 is a putative repressor gene with a repressor domain whose role in pituitary development is thought to repress downstream target genes. The extinction of Hesx1 is important for the activation of Prophet of Pit-1 (Prop1), which encodes a pituitary-specific paired-like homeodomain activator. It is postulated that Hesx1 and Prop1 function as opposing transcription factors and that the carefully regulated temporal sequence of their expression is essential for normal pituitary development. The pituitary phenotype in HESX1 mutations has been reported to be associated with: agenesis of the corpus callosum, hypoplasia of the optic nerves, pituitary hypoplasia and ectopic posterior pituitary. In other cases, MR imaging has evidenced unilateral hypoplasia of the optic nerve associated with normal or hypoplastic pituitary gland in patients with isolated GH deficits or with panhypopituitarism; pituitary gland aplasia has also been reported in HESX1 mutation. This is the first study on HESX1 expression in humans during adult life and it suggests that HESX1 in human adults is not turned off as in mice. These findings provide evidence that HESX1 might play a role in the maintenance of anterior pituitary cell types and function, as well as in the differentiation of pituitary adenomas. The apparent biphasic pattern of HESX1 with early expression during embryonic life, subsequent silencing and restored expression in the adult pituitary is fascinating, although the mechanism still needs to be better elucidated. 


\section{Common mutations of $\beta$-catenin in adamantinomatous craniopharyngiomas but not in other tumors originating from the sellar region}

Buslei R, Nolde M, Hofmann B, Meissner S, Eyupoglu IY, Siebzehnrubl F, Hahnen E, Kreutzer J, Fahlbusch R Department of Neuropathology, Friedrich Alexander University Erlangen-Nuremberg, Erlangen, Germany rolf.buslei@neuropatho.imed.uni-erlangen.de

Acta Neuropathol (Berl) 2005;109:589-597

Background: Although craniopharyngiomas are histologically benign, the recurrence rate is high. Two histological types have been recognized, papillary and adamantinomatous craniopharyngiomas, the latter being more frequent in the pediatric age. The survival expansion of normal neural stem cells in the nervous system depends on the activity of highly conserved developmental control pathways including the WNT and Hedgehog pathways. The significance of WNT/wingless pathway alterations in the pathogenesis of malignant tumors has been confirmed and craniopharyngiomas harboring $\beta$-catenin gene mutations (signaling cascade of WNT) have recently been reported.

Methods: Surgical specimens from patients with pituitary adenomas $(\mathrm{n}=60)$, craniopharyngiomas $(\mathrm{n}=41)$, Rathke's cleft cysts $(\mathrm{n}=10)$, arachnoidal cysts $(\mathrm{n}=8)$ and xanthogranulomas $(\mathrm{n}=6)$ were examined by immunohistochemistry and with mutational analysis for the expression pattern of $\beta$ catenin.

Results: $\beta$-Catenin mutations were found in $77 \%$ of craniopharyngiomas, all within the adamantinomatous subtype. All mutations affected exon 3 , which encodes the degradation targeting box of $\beta$-catenin, compatible with an accumulation of nuclear $\beta$-catenin. In addition, a novel 81-bp deletion of this exonic region was detected in one case. Immunohistochemical analysis confirmed a shift from membrane-bound to nuclear accumulation of $\beta$-catenin in $94 \%$ of the adamantinomatous tumors. Aberrant distribution patterns of $\beta$-catenin were not found in any of the other conditions.

Conclusion: $\beta$-Catenin mutations and/or nuclear accumulation were frequently and exclusively associated with the adamantinomatous variant of craniopharyngioma, which is histologically and genetically distinct from the papillary form and other sellar tumors.

Craniopharyngiomas occur in the sellar region comprising $3 \%$ of all intracranial tumors. Differential diagnosis between craniopharyngioma, Rathke's cleft cyst and xanthogranuloma is often difficult and genetic analyses might be helpful in the diagnostic work-up. Recent studies suggest that mutant $\beta$-catenin gene in craniopharyngiomas and pituitary adenomas may contribute to their tumorigenesis. This study shows that $\beta$-catenin gene mutations are found in all the adamantinomatous, but in none of the other sellar lesions; this is in contrast with recent reports suggesting that pituitary adenomas also carry such mutations. The information from this study contributes to an understanding of the pathogenesis of this devastating local tumor. The analysis of the functional importance of these pathways may lead to a better knowledge of brain tumor biology and may eventually result in the development of novel more mechanism-related therapies that would prevent the high relapse rate in these tumors and allow for more conservative surgery with less hypothalamic damage.

\section{Variability of isolated autosomal-dominant GH deficiency (IGHD II): impact of the P89L GH mutation on clinical follow-up and GH secretion}

Salemi S, Yousefi S, Baltensperger K, Robinson IC, Eble A, Simon D, Czernichow P, Binder G, Sonnet E, Mullis PE Paediatric Endocrinology, University Children's Hospital, Inselspital, Switzerland

Eur J Endocrinol 2005;153:791-802

Background: IGHD type 2 is inherited as an autosomal-dominant condition, and is mostly the result of splice site mutations in intron III (IVS3) of the GH-1 gene. Patients present with marked short stature, and respond well to exogenous GH treatment with no formation of antibodies. Among the various reported GH-1 gene mutations, however, the phenotype appears widely variable. This study aimed to analyze the impact of specific missense mutated P89L GH on clinical phenotype and at the cellular level.

Methods: Twelve subjects from 4 families presenting with P89L GH were clinically compared with 17 subjects from 5 families with the R183H GH missense mutation. Further, co-localization of the wildtype and mutant GH forms was studied in AtT-20 cells mouse pituitary gland by applying quantitative 
confocal microscopy analysis. Using immunofluorescent techniques, cells were double stained for GH and one of the following markers: endoplasmic reticulum (anti-Grp94), Golgi (anti- $\beta C O P$ ) and secretory granules (anti-Rab3a). In addition, GH secretion and cell viability were analyzed in detail. MRI of the pituitary gland was carried out.

Results: Eight of the patients carrying the P89L GH mutation showed additional anterior pituitary hormone deficiencies including TSH and ACTH, as well as reduction of anterior pituitary size. The two missense mutations showed different impacts on GH secretion from endocrine cell lines. Co-staining with antibodies against GH and either Grp94, anti- $\beta C O P$ or anti-rab3a revealed that wild-type GH and the two different mutations presented different subcellular localization. Mutant proteins may have a different impact on the secretory pathway, possibly caused by different folding or aggregation problems which are necessary for sorting, packaging and/or secretion through the regulated secretory pathway.

Conclusion: These findings showed that mutations leading to the autosomal-dominant IGHD type 2 may have a different impact on clinical features. Additional anterior pituitary hormone deficiencies and reduction of pituitary size can develop over time. The mechanism by which different missense isoforms may lead to different endocrine features is explained in this rare condition.

Autosomal-dominant isolated GH deficiency (IGHD) is a rare disorder that is commonly believed to be due to heterozygous mutations in the $\mathrm{GH}-1$ gene $(\mathrm{GH}-1)$. These splice site mutations lead to the production of two alternatively spliced GH molecules, 20- and 17.5-kDa hGH. The 17.5-kDa form of hGH has a dominant negative effect preventing the secretion of the normal wild-type 22-kDa hGH with a consequent deleterious effect on pituitary somatotrophs. In a murine model of this dominant negative mutation, there is evolution of the phenotype with later failure of PRL, TSH and gonadotrophin secretion. Long-term follow-up of 57 patients from 19 families with type II IGHD showed that evolving pituitary hormone deficiencies involving thyroid and adrenal functions may be a feature of this condition. Additionally, patients carrying one of the four missense mutations (R77C, R183H, P89L and V110F) implicated in IGHD type II have a normal GH-1 allele but are unable to secrete the normal form of GH in appropriate concentrations. This study provides an explanation for a range of clinical features associated with $\mathrm{GH}$ missense isoforms leading to progressive acquired pituitary disease. Similarly, impaired trafficking or degradation of mutated vasopressin proteins with progressive loss of vasopressin-producing neurons has been reported in familial autosomal-dominant central diabetes insipidus. The identification of these mechanisms and their role calls for more than just a description of new, affected patients and stresses the need for long-term clinical surveillance in IGHD II patients for the possible development of other multiple pituitary hormone deficiencies.

\section{Changes in expression of hypothalamic releasing hormone receptors in individual rat anterior pituitary cells during maturation, puberty and senescence}

Senovilla L, Garcia-Sancho J, Villalobos C

Instituto de Biologia y Genetica Molecular, Universidad de Valladolid, Valladolid, Spain

Endocrinology 2005; 146:4627-4634

Background: The anterior pituitary gland is composed of specialized cells that secrete multiple hormones including GH, FSH, LH, ACTH, and prolactin (PRL). The secretion of these hormones is under the stimulatory influence of specific hypothalamic releasing hormones (HRH). It was previously thought that each cell secretes only one pituitary hormone. It has however been reported that each cell can store more than one hormone. Furthermore, it has been shown that changes in expression of pituitary receptors for HRH are involved in critical endocrine changes that occur during life, such as postnatal development, puberty and aging. It has also been shown that multiple HRH receptors can be present in a single pituitary cell. This paper reports the expression of four HRH receptors in individual rat anterior pituitary cells throughout the whole lifespan.

Methods: The anterior pituitary of male and female Wistar rats of 0, 7, 15, 30, 45, 60, 90 and more than 540 postnatal days were removed and the cells dispersed. The responses of single cells to HRSs were assessed for changes in cytosolic calcium, measured by digital imaging fluorescence microscopy. 
Results: Three different cell pools were identified: (1) monoresponsive cells, holding a single class HRH receptor; (2) multiresponsive cells, and (3) non-responsive cells. Non-responsive cells were abundant at birth, multiresponsive cells were abundant at puberty, and monoresponsive cells dominated at senescence. The relative abundance of each HRH receptor changed largely with age but not by gender.

Conclusion: Anterior pituitary hormone secretion changes across life. This paper outlines a comprehensive characterization of changes in functional responses to CRH, LHRH, TRH and GHRH in individual male and female rat anterior pituitary cells throughout the lifespan. These changes are an expression of pituitary plasticity and probably contribute to endocrine changes observed during the lifespan.

This technical paper points to what could be a significant progress toward understanding pituitary plasticity. It adds a significant level of complexity to our generally simplistic view of 'one hypothalamic releasing hormone', one pituitary cell type. The multiresponsive cells are present at birth and contribute variably to anterior pituitary cell subpopulations, depending on the age. They tend to increase during maturation and puberty but decrease very much at senescence. These changes are the expression of pituitary plasticity and are likely to contribute to endocrine changes observed during the lifespan. This is a good example of the need to integrate systems biology and biological networks in endocrine physiology.

\section{Perinatal glucocorticoid treatment produces molecular, functional, and morphological changes in the anterior pituitary gland of the adult male rat}

Theogaraj E, John CD, Christian HC, Morris JF, Smith SF, Buckingham JC

Department of Cellular and Molecular Neuroscience, Division of Neuroscience and Mental Health, Imperial College London, Hammersmith Campus, London, UK

Endocrinology 2005;146:4804-4813

Background: It is generally accepted that adverse events in early life or during fetal development may have long-term sequelae on developing organisms and increase susceptibility to disease in later life. Stress or glucocorticoid treatment in perinatal life can induce long-term changes in the sensitivity of the hypothalamo-pituitary-adrenocortical (HPA) axis to the feedback actions of glucocorticoids and, hence, in glucocorticoid secretion as well. Annexin 1 (ANXA1) is one of the factors involved in mediating the regulatory effects of glucocorticoids on ACTH secretion at the pituitary level. This study tested the hypothesis that the programming actions of glucocorticoids on the HPA axis might include disruption of ANXA1-dependent mechanisms. The morphology of non-endocrine folliculostellate cells (FS) and corticotropes was also studied.

Methods: Dexamethasone was administered to pregnant or lactating rats via drinking water. The pituitary glands of their offspring were examined ex vivo at postnatal day 90.

Results: ANXA1 expression was reduced after both treatments, while IL-6 expression increased. Pituitary tissue from untreated control rats responded to dexamethasone with an increase in cell surface ANXA1 and a reduction in forskolin-induced ACTH release while pituitary tissue from dexamethasonetreated animals did not show this same response. Prenatal dexamethasone treatment reduced the size but not the number of FS cells, and caused a marked reduction in corticotroph number.

Conclusion: This study shows that prenatal or early postnatal glucocorticoid treatment exerts profound effects on adult pituitary gland morphology and function.

Abundant evidence supports the concept that adult diseases may have their origin in perinatal life. A correlation between low birth weight and increased risk of metabolic and cardiovascular disorders has been reported by several investigators. It has recently been suggested that elevated cortisol concentrations might represent a link between low birth weight and insulin resistance. Furthermore, the function of the HPA axis can be permanently programmed during development. The molecular mechanisms underlying the effects of perinatal insults on susceptibility to disease in adult life are gradually becoming clearer. This paper contributes to this line of evidence by showing that glucocorticoid treatment in the perinatal period permanently affect the number and morphology of corticotrophs, as well as the function of the HPA axis. 


\section{An assessment of petrosal sinus sampling for localization of pituitary microadenomas in children with Cushing's disease}

Batista D, Gennari M, Riar J, Chang R, Keil MF, Oldfield EH, Stratakis CA

Section on Endocrinology and Genetics, Developmental Endocrinology Branch, National Institute of Child Health and Human Development, National Institutes of Health, Warren G. Magnuson Clinical Center, Bethesda, Md., USA

J Clin Endocrinol Metab 2006;91:221-224

Background: ACTH-secreting adenomas are the second most frequent pituitary adenomas in children and adolescents after prolactinomas. ACTH-secreting adenomas are the most frequent cause of endogenous Cushing's syndrome in children. Pituitary corticotropinomas in children are usually small and MRI studies often fail to detect the tumor. This study investigated the use of bilateral inferior petrosal sinus sampling (BIPSS) as a tool for localization of a pituitary adenoma.

Methods: A retrospective review of the records of 141 children admitted for evaluation of Cushing's disease (CD) from 1982 to 2004 at the National Institute of Health. 94 children (49 males and 45 females, age $13 \pm 3.2$ years) met the criteria for study admission: (1) consistently elevated urinary free cortisol levels before treatment with normalization after transsphenoidal surgery (TSS), and (2) a negative preoperative pituitary MRI or consistently negative imaging studies throughout their evaluations, or positive MRI but inconsistent biochemical data. The patients underwent bilateral central and peripheral simultaneous blood sampling for measurement of ACTH before and after intravenous administration of oCRH.

Results: Localization of a microadenoma by BIPSS was in agreement with surgical location in only $58 \%$ of cases. This figure did not improve with the combined use of BIPSS and MRI. The procedure was safe and completely effective in all patients.

Conclusion: BIPSS is rarely, if ever, needed in children and is not a reliable test for lateralization of a pituitary adenoma.

As $C D$ is so rare in children, we tend to follow our adult endocrinologists' and neurosurgical colleagues' routines. This paper reminds us that pediatric $C D$ is a different entity. In the 11- to 15-year age range, ACTH-secreting adenomas are the most frequent cause of adrenal hyperfunction and the second most frequently occurring pituitary adenoma after prolactinomas. A macroadenoma is rarely the cause of $C D$ in children. Clinical presentation is highly variable, with signs and symptoms that can range from subtle to obvious. The diagnosis is generally delayed since a decrease in growth rate may be the only symptom in a long time period. The differential diagnosis of CD includes adrenal tumors, ectopic ACTH production, and ectopic CRH-producing tumors. However, ectopic ACTH secretion is extremely rare in the pediatric age. In a child/adolescent with suspected $C D$, the diagnosis is based on measurement of basal and stimulated levels of cortisol and ACTH. Measurement of 24-hour urinaryfree cortisol is elevated, and a low dose of dexamethasone $(15 \mu \mathrm{g} / \mathrm{kg})$ at midnight does not induce suppression of morning serum cortisol concentrations as in normal subjects. Suppression of spontaneous circadian variations in serum cortisol is another feature of CD. Suppression of cortisol by more than $50 \%$ after a high dose of dexamethasone $(150 \mu \mathrm{g} / \mathrm{kg})$ given at midnight will confirm that hypercortisolism is due to an ACTH-secreting pituitary adenoma. All patients should undergo pituitary MRI with the administration of gadolinium, but since ACTH-secreting pituitary adenomas are significantly smaller than all other types of adenomas, often with a diameter of $\leq 2 \mathrm{~mm}$, pituitary MRI may fail to visualize the tumor. In most instances the diagnosis of CD can be made from initial clinical and laboratory data. Bilateral inferior petrosal sinus sampling has a high specificity, guaranteeing that no patient with extrapituitary Cushing's syndrome runs the risk of being submitted to transsphenoidal surgery, but it gives a significant number of false negative results. The study of Batista et al. confirms that BIPSS is of limited use in the diagnosis of pediatric CD. 


\section{Hypothalamic 3',5'-cyclic adenosine monophosphate response element-binding protein loss causes anterior pituitary hypoplasia and dwarfism in mice}

Mantamadiotis T, Kretz O, Ridder S, Bleckmann SC, Bock D, Grone HJ, Malaterre J, Dworkin S, Ramsay RG, Schutz G Molecular Biology of the Cell, Deutsches Krebsforschungszentrum, Heidelberg, Germany theo.mantamadiotis@petermac.org Mol Endocrinol 2006;20:204-211

Background: A number of transcription factors regulate GHRH and GH gene expression. The cAMP response element (CRE)-binding protein (CREB) is one of the first transcription factors shown to have an impact upon somatic growth. The generation of mice with total or tissue-specific loss of CREB has revealed the importance of this transcription factor in normal physiology. This study addresses the role of CREB in the brain in CREB-transgenic mice in whom CREB was specifically deleted in the brain but not in the pituitary gland.

Methods: Generation of conditional CREB mutant mice and analysis of pituitary morphology, pituitary hormones and IGF-I expression and expression of hypothalamic factors.

Results: Loss of CREB in the brain but not in the pituitary results in reduced postnatal growth consistent with GH-dependent dwarfism. Reduction of the amount of GHRH peptide.

Conclusion: This study shows that brain-specific CREB loss resulted in a phenotype similar to that found in the pituitary-specific dominant-negative CREB transgenic mouse. CREB is a key transcription factor that regulates somatic growth at multiple levels in the hypothalamic-GH-IGF axis.

Several transcription factors participate in pituitary development and in the regulation of the transcription of specific pituitary genes. However, the transcription factors involved in hypothalamic growth regulation have been less well studied. CREB is an important transcription factor with many functions in the CNS, and is required for efficient pituitary somatotroph proliferation and function in vivo and in vitro. Loss of CREB in the pituitary causes pituitary hypoplasia and severe dwarfism. This study shows that loss of CREB in the hypothalamus causes a similar phenotype. These results provide a new model for the study of GHRH function and could possibly generate new research in children with IGHD. This paper will probably start a race among geneticists to identify mutations of the Creb1 gene in syndromes affecting human somatic growth.

\section{The role of CBP/p300 interactions and Pit-1 dimerization in the pathophysiological mechanism of combined pituitary hormone deficiency}

Cohen RN, Brue T, Naik K, Houlihan CA, Wondisford FE, Radovick S

Section of Pediatric and Adult Endocrinology, Department of Medicine, University of Chicago, Chicago, III., USA roncohen@medicine.bsd.uchicago.edu

J Clin Endocrinol Metab 2006;91:239-247

Background: Pit-1 is reported to regulate GH, PRL, TSH $\beta$-subunit, and Pit-1 genes by binding to response elements present in their promoter regions and recruiting coactivator proteins to the transcriptional complex. The cAMP response element-binding protein (CREB)-binding protein (CBP)/p300 coactivator class has been implicated as important in this process. Patients may have either an inherited autosomal recessive or an autosomal-dominant disorder caused by a truncated Pit- 1 molecule that is unable to bind to DNA while others have normal DNA bindings. The objective of this study was to clarify the mechanism by which mutant PIT-1 molecules with intact DNA binding are unable to activate target genes.

Methods: Five human Pit-1 molecules were chosen for a study based on their predicted or reported normal DNA-binding characteristics. DNA-binding and functional studies were used to assess the following five specific PIT-1 mutations: F135C, R143Q, A158P, K216E, and R271W.

Results: Wild-type Pit-1 bound to both elements from the GH and PRL genes as either a monomer or a dimer. In gel-shift studies using radiolabeled DNA-binding elements from GH and prolactin genes, the 
K126E mutant displayed markedly enhanced Pit-1 dimer binding to either element, whereas the R271W mutant bound with high avidity, but only as a monomer. On the contrary, the R143Q mutant had no detectable DNA binding on either element and the F135C and A158P mutants displayed nearnormal DNA-binding characteristics. CBP/p300 bound poorly to the A158P and K216E mutant Pit-1 molecules, but bound normally to the F135C, R143Q, and R271W mutants. In functional assays, $\mathrm{CBP} / \mathrm{p} 300$ cotransfection with mutant Pit-1 expression vectors resulted in less transactivation of either $\mathrm{GH}$ or prolactin reporter genes.

Conclusion: This study demonstrated that both Pit-1 dimerization and CBP/p300 interactions in Pit-1dependent gene activation are crucial. Pit-1 dimerization is not sufficient for target gene activation unless CBP/p300 is also bound to Pit-1. DNA binding is essential for Pit-1 action in the pituitary.

Pituitary-specific transcription factor-1 is a member of the POU transcription factor family, whose expression is restricted to the anterior pituitary. Pit-1 is the major cell-specific activator of hormone expression from somatotrophs and lactotrophs and Pit-1 protein is expressed in caudomedial thyrotrophs. Pit-1 usually binds to multiple sites on target genes, and dimerization of Pit-1 on DNA elements is important for high-affinity DNA bindings. Naturally occurring mutations in Jackson and Snell dwarf mice are associated with GH, PRL and TSH deficiencies. Although the hormonal phenotype of PIT-1 mutations described in humans is similar among these patients, the inheritance pattern and phenotypic presentation are different, suggesting different location and functional perturbations caused by the mutation. CREB-binding proteins (CBP) and p300 are essential transcriptional coactivators for a large number of regulated DNA-binding transcription factors, including CREB, nuclear receptors, and STATs. This study provides direct evidence for several important mechanisms involved in the pathogenesis of combined pituitary hormone deficiencies. It also highlights the role of the CAMP response element-binding protein CREB/p300 interactions and Pit-1 dimerization in this complex process.

\section{Pituitary-specific Gata2 knockout: effects on gonadotrope and thyrotrope function}

Charles MA, Saunders TL, Wood WM, Owens K, Parlow AF, Camper SA, Ridgway EC, Gordon DF

Department of Human Genetics, University of Michigan, Ann Arbor, Mich.; Division of Endocrinology,

University of Colorado, Aurora, Colo., and National Hormone \& Peptide Program,

Harbor-UCLA Medical Center, Torrance, Calif., USA

Mol Endocrinol 2006 [Epub ahead of print]

Background: GATA transcription factors are emerging as critical players in mammalian reproductive development and function. More specifically, GATA2 is expressed in the developing pituitary gland. High levels of GATA-2 lead to gonadotrope lineage whereas GATA-2 in the presence of Pit-1 will determine thyrotrope lineage. Knowledge about the role of these transcription factors is still in its infancy. Methods: A pituitary-specific knockout of Gata2, designed so that the DNA-binding zinc-finger region is deleted in the presence of a pituitary-specific Cre recombinase transgene, was generated.

Results: The immunohistochemistry of the pituitary gland in Gata2 knockout newborn mice showed that the number of POMC- and GH-immunoreactive cells was indistinguishable between mutant and wildtype pituitary glands. Few FSH and TSH immunostaining-positive cells were found in the mutant Gata2 mice. Male Gata2-deficient mice exhibited growth delay from 3 to 9 weeks of age, and adult mutants produced less thyrotropin in response to severe hypothyroidism following radiothyroidectomy. These mice showed reduced secretion of gonadotropins basally and in response to castration challenge. The reduced level of gonadotropins was associated with normal testes size and did not preclude fertility. Gata2-deficient mice exhibited elevated levels of Gata3 transcripts in the pituitary gland.

Conclusion: Gata2 has a role in gonadotrope and thyrotrope cell development and function. Gata3 is likely to be cooperating to maintain thyrotrope and gonadotrope function.

In mammals, the GATA family is composed of six members divided into two families based on location, structure, and function. In particular, GATA-1/2/3 members are most often though not exclusively associated with hematopoietic cell lineages and nervous system development function. Previous studies showed that Pit-1 functionally cooperates with Gata2 to stimulate transcription of the TSH 
$\beta$ gene. Pit-1 and Gata2 are uniquely co-expressed in pituitary thyrotropes and activate transcription by binding to a composite promoter element. The identification of the role of Gata2 represents a further step forward in the understanding of the complex signaling pathway involved in thyroid and reproduction function. Rapid advances will most likely be made when animals with Gata gene ablation in specific tissue are generated. If you were looking for additional links between the brain, the pituitary and obesity, it is worth mentioning that GATA2 (and GATA3) are specifically expressed in preadipocytes and that their downregulation sets the stage for terminal differentiation. Constitutive GATA2 (and GATA3) expression suppressed adipocyte differentiation and trapped cells at the preadipocyte stage. This effect was mediated, at least in part, through the direct suppression of PPAR $\gamma$ and defective GATA2 and GATA3 expression is associated with obesity [1].

\section{Functional relationship between LHX4 and POU1F1 in light of the LHX4 mutation identified in patients with pituitary defects}

\section{Machinis K, Amselem S}

Institut National de la Sante et de la Recherche Médicale, Unité 654, Hôpital Henri Mondor, Créteil, France

J Clin Endocrinol Metab 2005;90:5456-5462

Background: A cascade of signaling molecules and transcription factors play significant roles in pituitary organogenesis. In particular, Lhx4 is a closely related gene to Lhx3 expressed throughout the invaginating pouch, with restriction of subsequent expression to the future anterior lobe. While Lhx4 mutant mice die shortly after birth, patients carrying Lhx4 mutation display combined pituitary hormone deficiency, structural hypothalamic pituitary abnormalities and Chiari I malformation. The precise mechanism underlying these discrepant phenotypes is not fully understood.

Methods: A candidate gene approach based on the patients' phenotypic features was used. Various LHX4 isoforms were expressed in Chinese hamster ovary cells and transfection experiments were performed. Results: The study shows that the POUF1 upstream sequence contains a putative human-specific LHX4 binding element, a gene encoding a POU homeodomain transcription factor already known as the main regulator of GH expression. Normal LHX4 recognizes, binds and activates the POUF1 regulatory sequence, whereas mutant LHX4 proteins display a marked decrease in their DNA-binding capacity. The mutant LHX4, however, does not impair normal LHX4 ability to activate the POUF1 regulatory sequence. Conclusion: These findings provide evidence for (1) a human-specific regulatory element in the POUF1 upstream regulatory sequence which confers responsiveness to LHX4 and (2) a molecular defect that impairs the capacity of LHX4 to bind and regulate the main activator of GH expression, i.e. POUF1.

The pathophysiology of combined pituitary hormone deficiency (CPHD) is just beginning to be elucidated, with mutations in genes encoding transcription factors expressed at different stages of pituitary development. Among them, two closely related genes, LHX3 and LHX4, are believed to share redundant biological properties. To date, there has been only one published report of a mutation of LHX4 [2]. The patient with LHX4 germline splice mutation displayed: CPHD (GH, TSH, ACTH; LH/FSH and PRL were not evaluated), anterior pituitary hypoplasia, ectopic posterior pituitary and extrapituitary manifestations that included a poorly formed sella turcica, a persistent craniopharyngeal canal and pointed cerebellar tonsils suggestive of Chiari malformation. The authors nicely demonstrate the absence of a dominant-negative effect of the mutant LHX4 proteins over the normal ones and suggest that haploinsufficiency of normal LHX4 would account for the GH defect of the patient. This is a further step forward in the understanding of the complex signaling pathway involved in pituitary organogenesis.

\section{Role of Prophet of Pit-1 (PROP1) in gonadotrope differentiation and puberty}

Vesper AH, Raetzman LT, Camper SA

Department of Human Genetics, University of Michigan, Ann Arbor, Mich., USA

scamper@umich.edu

Endocrinology 2006;147:1654-1663

Background: Puberty and fertility depend on the maturation of the hypothalamic-pituitary-gonadal axis. The pituitary, driven by the pulsatile release of $\mathrm{GnRH}$ from the hypothalamus, secretes gonadotropins 
which stimulate gonadal steroidogenesis and gametogenesis. The differentiation of a gonadotropin-specific cell lineage during fetal development is under the coordinated regulation of a host of transcription factors. Prop1 (Prophet of Pit-1) encodes a paired-like homeodomain transcription factor required for pituitary organogenesis. Mutations of the Prop1 gene cause growth insufficiency, hypothyroidism, adrenal insufficiency and infertility in rodents and humans and are the most common cause of multiple pituitary hormone deficiency (MPHD). In this report, transgenic mice that persistently overexpress Prop1 were generated in order to study the influence of Prop1 on gonadotropes and puberty.

Methods: Prop1 transgenic mice were generated and bred for seven generations. Their pituitary cell populations were examined by immunochemistry. Pituitary gene expression was determined by microarray analysis and quantitative RT-PCR. The studies were performed on embryos and postnatally at different age points.

Results: Transgenic mice overexpressing the Prop1 gene in gonadotropes and thyrotropes show delayed puberty, transient hypogonadotropic hypogonadism, and transient growth insufficiency. They recover from these abnormalities and exhibit normal size and fertility at 3 months. No abnormalities in the expression of the transcription factors EGR1, NR5A1, GATA2, TBX19, NR0B1, or of TGF- $\beta$ pathway members, including activin, inhibin and activin receptors, were found. Microarray analysis identified the inhibitory transmembrane receptor gene Klrg1 and the protease gene prss 21 as as candidates involved in gonadotrope development.

Conclusion: The authors conclude that variations in Prop1 expression could affect growth spurt and the onset of puberty in humans.

Pituitary development, differentiation and function is under the regulation of a complex network of transcription factors, and, ultimately, the secretion and function of hypothalamic releasing and inhibiting hormones. The action of these hypothalamic hormones is, in turn, also modulated by a host of transcription factors that regulate the transcription of specific genes. Prop 1 is one of the transcription factors crucial for pituitary development and function. Through the use of transgenic mice, this elegant study provides indirect evidence that variations in Prop1 expression may also be involved in disorders of growth and in the onset of puberty, so far in the rodent only.

\section{An essential role for the hematopoietic transcription factor lkaros in hypothalamic-pituitary-mediated somatic growth}

Ezzat S, Mader R, Fischer S, Yu S, Ackerley C, Asa SL

Department of Medicine, Mount Sinai Hospital, University of Toronto, Toronto, Ont., Canada

Proc Natl Acad Sci USA 2006;103:2214-2219

Background: Ikaros is a transcription factor originally described as involved in the regulation of genes expressed in lymphoid cells, and subsequently found to be expressed in the mouse pituitary. This study investigated the direct role of Ikaros (IK) in hypothalamic-pituitary-mediated somatic growth.

Methods: Rat mammotroph GH4 cells stably expressing IK1 were transfected with the 5'-GHRH-R or GHRH promoter. Expression of endogenous GHRH was determined by Western blotting and circulating GHRH was measured. IK-null mice were generated and morphologic and immunohistochemical studies in fetal and neonatal brains were carried out and serial sections of the hypothalamus and the pituitary were analyzed. The quantitative morphology of the pituitary was assessed by using a light microscope and microcomputer imaging software. Hormone measurements of IGF-I, PRL, ACTH and TSH were assessed. GH treatment of IK-deficient mice was performed for 6 weeks. Hematopoietic cell repopulation was evaluated in bone marrow recipients.

Results: Ikaros-null mice displayed a GH-deficient phenotype with progressive postnatal growth failure associated with low IGF-I in the absence of TSH and PRL deficiencies, but with ACTH defect. Autopsy revealed reduced pituitary and adrenal glands. GH administration was followed by sustained postnatal growth and increased IGF-I concentration, whereas bone marrow reconstitution was not. Morphometric evaluation showed a reduction in GH staining. Ikaros was identified in wild-type hypothalamic arcuate nuclei, where it colocalized with GHRH in cell bodies, while GHRH immunoreactivity was undetectable. Finally, Ikaros regulated GHRH gene expression and enhanced GHRH promoter activity. Conclusion: Ikaros is involved in the regulation of the somatotropic axis through a neuroendocrine GHRH-mediated mechanism. Ikaros has a critical role in neuroendocrine system machinery. 
Several transcription factors are implicated in pituitary development and expression of tissue-specific genes. Increasing evidence indicates that the endocrine and immune systems are functionally integrated to coordinate stress response. Ikaros proteins are zinc finger transcription factors involved in lymphopoietic cell differentiation and immune functions. Mutations of the Ikaros gene have been found in infants with acute lymphoblastic leukemia and a recent study reports that the lkaros gene is crucial in the development of the pituitary and adrenal glands. Ikaros-deficient mice, in fact, die because of adrenocortical insufficiency and can be cured by glucocorticoid replacement. This study investigated the direct role of Ikaros in the development and function of the pituitary-somatotroph axis. It shows a direct involvement of the Ikaros gene in the development and function of pituitary somatotropes. Ikaros was found to be expressed in the hypothalamic nuclei where it colocalized with GHRH in cell bodies and regulates its expression. Furthermore, loss of Ikaros impaired pituitarydirected $\mathrm{GH}$ and adrenocortical function; GH administration was followed by sustained growth hormone effects. Pituitary GH-dependent function in Ikaros-null mice is not reversed by wild-type lymphocyte reconstitution. The identification of Ikaros as a factor involved in the regulation of the GHRH-IGF-I axis provides new insights into the transcriptional mechanisms which interface the neuroendocrine and immune systems.

\section{Reviews}

\section{Does growth hormone cause cancer?}

Jenkins PJ, Mukherjee A, Shalet SM

Departments of Endocrinology, St Bartholomew's Hospital, London, UK

Clin Endocrinol (Oxf) 2006;64:115-121

Background: Growth hormone has potential cancer-enhancing properties. A body of evidence in support of this hypothesis comes from in vitro and animal studies, epidemiological observation within the general population, patients with GH deficiency or excess and therapeutic manipulations of GH and IGF-I action. Methods: This is a comprehensive review.

Results: Epidemiological studies of patients with acromegaly indicate an increased risk of colorectal cancer, although risk of other cancers is unproven. A long-term follow-up study of children deficient in GH who were treated with pituitary-derived $\mathrm{GH}$ has indicated an increased risk of colorectal cancer. Conversely, extensive studies of the outcome of GH replacement in childhood cancer survivors show no evidence of an excess of de novo cancers, and more recent surveillance of children and adults treated with $\mathrm{GH}$ has revealed no increase in observed cancer risk.

Conclusion: Given the experimental evidence indicating that GH/IGF-1 produces an antiapoptotic environment that may favor survival of genetically damaged cells, long-term surveillance is necessary over a long time period since even a subtle alteration in the environmental milieu of this sort, although not directly inducing cancer, could result in acceleration of carcinogenesis. In any case, even if patients who undergo GH/IGF-1 therapy do show a small increase of cancer risk compared to untreated patients with GH deficiency, it is likely that their rate of risk will be the same as that found in the general population. A growth/metabolic recovery with the establishment of normality will need to be weighed against the known morbidity of untreated GH deficiency.

This review provides a comprehensive description of experimental and clinical studies on the cancerenhancing effect of growth hormone. The authors conclude that although there is no definitive convincing evidence that growth hormone per se is absolutely safe, careful long-term surveillance programs in GH-treated patients are needed. 


\section{Efficacy and safety of exogenous melatonin for secondary sleep disorders and sleep disorders accompanying sleep restriction: meta-analysis}

Buscemi N, Vandermeer B, Hooton N, Pandya R, Tjosvold L, Hartling L, Vohra S, Klassen TP, Baker G

University of Alberta/Capital Health Evidence-Based Practice Centre, Department of Pediatrics,

University of Alberta, Edmonton, Alta, Canada

nina.buscemi@ualberta.ca

BMJ 2006;332:385-393

Background: Sleep disorders affect a high number of subjects in industrialized countries. One of the most commonly used treatments in sleep disorders is melatonin. The efficacy and safety of exogenous melatonin in managing secondary sleep disorders and sleep disorders accompanying sleep restriction, such as jet lag and shift work disorder, has been reported to be substantially heterogeneous.

Methods: A systematic review of full-text articles deemed relevant was evaluated independently by two reviewers. Randomized controlled trials involving human participants were retrieved to assess the efficacy of melatonin, whereas the safety review included both randomized and non-randomized trials. The data extrapolated included sleep onset latency, sleep efficiency, sleep quality, wakefulness after sleep onset and total sleep time. Secondary sleep disorder was defined as when the disorder was likely to be the cause, while sleep restriction was defined as when participants were subject to transmeridian air travel or other sleep schedule alteration. The randomized controlled trials were assessed by using the Jadad Scale and criteria by Schulz et al., and the non-randomized controlled trials were assessed by the Downs and Black checklist. One reviewer extracted data and another reviewer verified the data extracted. The inverse variance method was used to weight studies and the random effects model was used to analyze data.

Results: Six randomized controlled trials with 97 participants showed that melatonin does not have a significant effect on sleep onset latency in people with secondary sleep disorders [weighted mean difference -13.2 (95\% confidence interval -27.3 to 0.9 ) $\mathrm{min}$ ]. Nine randomized controlled trials with 427 participants showed no evidence that melatonin had an effect on sleep onset latency in people who had sleep disorders accompanying sleep restriction [-1.0 (-2.3 to 0.3) min]. Seventeen randomized controlled trials with 651 participants showed no differences in adverse effects between placebo or melatonin with short-term use ( $\leq 3$ months).

Conclusion: Melatonin does not affect either sleep onset latency or sleep efficiency in treating secondary sleep disorders or sleep disorders accompanying sleep restriction, such as jet lag and shift work disorder. Short-term use of melatonin is safe.

Melatonin ( $\mathrm{N}$-acetyl-5-methoxytryptamine) is a hormone produced by the pineal gland in the brain in response to darkness. It is made available when tryptophan is converted to serotonin and then enzymatically converted to melatonin in the pineal gland. Serum levels are low during the day, with peak levels occurring from 2 to 4 a.m. The key enzyme in melatonin synthesis is $\mathrm{N}$-acetyltransferase (NAT) whose activity increases during darkness. This explains why melatonin is mainly secreted at night. The circadian rhythm of melatonin synthesis is controlled by the suprachiasmatic nuclei - the 'biologic clock'. This clock itself functions rhythmically, being entrained by environmental synchronizers. Exogenous melatonin has been reported to synchronize some circadian rhythms, such as body core temperature, aging, blindness, shift work, night work, phase-advanced or phase-delayed sleep syndrome, and jet lag. This meta-analysis, however, shows that melatonin, although safe in the short run, has no efficacy in secondary sleep disorders and sleep restriction. Whether the observations of this review will have an impact on guiding clinicians and patients in treatment decisions for the management of sleep disorders remains to be seen. 


\title{
Human growth hormone-releasing factor (hGRF)1-29-albumin bioconjugates activate the GRF receptor on the anterior pituitary in rats: identification of CJC-1295 as a long-lasting GRF analog
}

\author{
Jette L, Leger R, Thibaudeau K, Benquet C, Robitaille M, Pellerin I, Paradis V, van Wyk P, Pham K, Bridon DP
}

Department of Research, ConjuChem Inc., Montreal, Que., Canada

Endocrinology 2005;146:3052-3058

Background: Growth hormone secretion is stimulated by hypothalamic GH-releasing factor (GRF). Human GRF is a 44 amino acid peptide that binds to specific receptors on the somatotroph cell surface. This neurohormone, in vivo, has a very short half-life. Several experiments have attempted to develop long-acting forms of the peptide, such as polyethylene glycol derivatives. Another useful tool for increasing the half-life of small molecules or peptides is in vivo bioconjugation to serum albumin. This study reports on the results of three $\mathrm{hGRF}_{1-29}$ analogs with the ability to conjugate to serum albumin. Methods: Three maleimido derivates of human $\mathrm{GRF}_{1-29}$ were synthesized and bioconjugated to human serum albumin ex vivo. All three human serum albumin conjugates were stable in vitro and were bioactive in a GH secretion assay. The GH-releasing activity in vivo was tested by administering the peptides in a single bolus injection subcutaneously $(1 \mu \mathrm{mol} / \mathrm{kg})$ to freely moving rats.

Results: All three hGRF analogs proved to be stable. In vitro studies showed that all three analogs stimulated GH secretion from cultured rat anterior pituitary cells in a dose-dependent manner. In addition, they all produced an acute secretion of GH when administered to freely moving rats. The GRF analogs were still detectable in serum in the nanomolar range $72 \mathrm{~h}$ after injection, whereas the concentrations of hGRF $_{1-29}$ disappeared after $1 \mathrm{~h}$.

Conclusion: The results of this study led to the identification of a hGRF analog, namely CJC-1295, as a stable and long-acting GH-releasing factor.

Isolated GH deficiency (GHD) can be due to abnormalities in the secretion or action of endogenous GRF. Most children with IGHD grow when treated with GRF, indicating normal functioning of the somatotroph cells. However, because of the short half-life of native GRF, the peptide needs to be administered in a pulsatile fashion. The potential ability of these hGRF analogs to elicit a sustained release of $\mathrm{GH}$ over time could be exploited for therapeutic use.

\section{New hopes: BMP-4 for Cushing's disease}

\section{Bone morphogenetic protein-4 inhibits corticotroph tumor cells: involvement in the retinoic acid inhibitory action}

Giacomini D, Paez-Pereda M, Theodoropoulou M, Labeur M, Refojo D, Gerez J, Chervin A, Berner S, Losa M, Buchfelder M, Renner U, Stalla GK, Arzt E

Laboratorio de Fisiologia y Biologia Molecular, Departemento de Fisiologia, Biologia Molecular y Celular, Facultad Ciencias Exactas y Naturales, Universidad de Buenos Aires, Ciudad Universitaria, Buenos Aires, Argentina

Endocrinology 2006;147:247-256

Background: Bone morphogenetic protein-4 (BMP-4), a member of the TGF- $\beta$ superfamily, plays a central role during pituitary organogenesis. In addition, it is overexpressed in prolactinomas models, including dopamine receptor- 2 knockout mice. The potential action of BMP-4 in pituitary corticotroph tumor growth was evaluated and the role of retinoic acid was also considered.

Methods: BMP-4 immunoreactivity and expression in pituitary corticotroph human tumors was studied. ACTH-secreting experimental tumors were formed by injecting clone cells (AtT-20 Smad-4dn cells) into nude mice. The antiproliferative effect of BMP-4 on tumor growth was evaluated by using a specific BMP-4 antagonist. 
Results: BMP-4 was found to be expressed in normal human pituitary corticotrophs whereas its expression was reduced in pituitary ACTH-secreting adenomas. BMP-4 treatment of nude mice that developed corticotrophinoma produced an inhibitory effect on ACTH secretion and cell proliferation. AtT-20 cells stably transfected with a dominant-negative form of the BMP-4 signal cotransducer Smad4 or the BMP-4 inhibitor noggin increased tumorigenicity in nude mice, showing that BMP-4 has an inhibitory role on corticotroph tumorigenesis in vivo. Retinoic acid induces both BMP-4 transcription and expression and its antiproliferative action is blocked in Smad-4dn- and noggin-transfected AtT-20 cells that do not respond to BMP-4.

Conclusion: The results of this study suggest that BMP-4 expression is reduced in pituitary ACTH-secreting adenomas. BMP-4 has a pivotal role in the regulation of corticotroph cell physiology and in the inhibition of its tumorigenic potential. BMP-4 induction by retinoic acid may be an interesting mechanistic potential target as a treatment option for Cushing's disease.

Corticotroph adenomas are small microadenomas accounting for $10-15 \%$ of clinically recognized pituitary adenomas. Although pluriclonality of transformed corticotroph cells has been suggested for the pathogenesis of Cushing's disease, monoclonal expansion appears to be one of the mechanisms involved in ACTH-secreting pituitary tumor development. This is the first study which shows that BMP-4 expression regulates not only corticotroph cell physiology but also inhibits both ACTH production and corticotroph cell proliferation; it is thus possible to hypothesize the involvement of BMP4 in corticotroph cell tumorigenesis. Indeed, BMP-4 acts as a regulator both of pituitary cells undergoing organogenesis differentiation and of transformed adult pituitary cells. So, the induction of BMP-4 transcription and expression in corticotrophs after retinoic acid treatment suggests that BMP-4 and retinoic acid may share a similar mechanism. Retinoic acid treatment has been proved to be successful in reducing proopiomelanocortin gene transcription and ACTH production by inhibiting transcriptional activity in pituitary ACTH-secreting cells. In vivo experiments in nude mice have also shown that retinoic acid administration completely blocks corticotroph tumor growth and reverses the endocrine alterations and symptoms of Cushing's disease. The inhibitory mechanism of BMP-4 represents a novel mechanism for an understanding of the pathogenesis of pituitary corticotroph cells. These data suggest that retinoic acid may be a potential therapeutic drug for the treatment of Cushing's disease. But do not prescribe it yet; you recall that RA dimerize with many other transcription factors, including some steroid hormone receptors without therapeutic effects.

\section{Expression and growth dependency of the insulin-like growth factor I receptor in craniopharyngioma cells: a novel therapeutic approach}

Ulfarsson E, Karstrom A, Yin S, Girnita A, Vasilcanu D, Thoren M, Kratz G, Hillman J, Axelson M, Larsson O, Girnita L Department of Neurosurgery, Institution for Surgical Sciences, Stockholm, Sweden

Clin Cancer Res 2005; 11:4674-4680

Background: Craniopharyngiomas account for $2-5 \%$ of all intracranial neoplasms and $5.6-13 \%$ of intracranial tumors in children. Despite their benign histological nature, their prognosis and outcome may be unfavorable due to the proximity of the tumor to vital structures and, in particular, to the hypothalamic-pituitary area. Tumor regrowth after partial removal varies among published reports between 63 and $90 \%$. Although GH treatment in children with craniopharyngioma is a part of the standard treatment, the possible role of IGF-1/IGF-I receptor-mediated tumor recurrence is still being debated. Methods: Primary cultures of human craniopharyngioma cells were isolated and appropriately prepared. Immunohistochemical analysis of tumor tissues for IGF-IR expression and IGF-I dependency was performed.

Results: Five of the craniopharyngioma cell lines exhibited a strong immunoreactivity to IGF-IR, whereas two of the cell lines showed no or very low expression of the receptor and were independent from IGF-I. Four of the cell lines with high IGF-IR expression were responsive to IGF-I alone or in combination with T3, but not to growth hormone, in agreement with the well-known growth stimulatory effects of IGF-I. Treatment with an IGF-IR inhibitor showed that cells with high IGF-IR expression were promptly responded promptly with decreased IGF-I-stimulated phosphorylation followed by growth arrest. These responses were not seen in cells with no/very low receptor expression. Growth of cell lines with low IGF-IR expression was only slightly affected by IGF-IR inhibition. 
Conclusion: Craniopharyngioma cells express IGF-IR in cell culture and IGF-I alone promotes tumor growth in some but not all patients. Indeed, inhibition of IGF-IR phosphorylation in cells with high IGF-IR expression results in marked reduction of cell line proliferation.

IGF-I is recognized to exert powerful effects on each of the key stages of cancer development, on the behavior of cell proliferation and apoptosis, on angiogenesis and metastasis, as well as possibly increasing resistance to chemotherapeutic agents. It is therefore natural to pose the question 'Do long-term survivors of childhood cancers including craniopharyngiomas who need GH treatment run an increased risk of tumor recurrence?' Indeed, there was no significant trend in relative risk of recurrence with time on GH treatment or with time elapsed since this treatment started as shown in a large study in 180 children with brain tumors [3]. Data from the Childhood Cancer Survivor Study are also consistent with the finding that GH does not increase the risk of recurrence for both primary brain tumors and acute leukemia [4]. On the other hand, other mechanisms including mutant $\beta$-catenin gene cells mutation, at least in the pediatric craniopharyngioma type, also contribute to tumorigenesis suggesting that the mechanism of tumor growth is rather complex. The findings of this study provide powerful support for the strategy of IGF-IR inhibitors in the treatment of craniopharyngioma. How such treatment can be balanced between GH promoting IGF-I generation and growth and the blockade of IGF-I action at its receptor remain puzzling.

\section{Food for thought}

\section{Gene expression profiling during cellular differentiation in the embryonic pituitary gland using cDNA microarrays}

Ellestad LE, Carre W, Muchow M, Jenkins SA, Wang X, Cogburn LA, Porter TE

Department of Animal and Avian Sciences, and Molecular and Cell Biology Program, University of Maryland,

College Park, Md., USA

Physiol Genomics 2006 [Epub ahead of print]

Background: Anterior pituitary cell types do not arise simultaneously but rather differentiate during embryogenesis in a temporally distinct behavior. A cascade of signaling molecules and transcription factors plays an important role in organ commitment, cell proliferation, cell patterning and terminal differentiation. It is likely that a large number of signaling molecules and transcription factors necessary for normal pituitary development are still unidentified. DNA microarrays technology may detect additional genes involved in pituitary gland cell differentiation.

Methods: Avian $\times$ Avian broiler chicken embryos were used. Anterior pituitary tissue was collected and the extracted RNA was quantified. Microarrays containing 5,128 unique cDNAs expressed in the chicken neuroendocrine system were produced and used to identify genes with potential involvement in the onset of thyroid-stimulating hormone $\beta$-subunit (TSH- $\beta$ ), growth hormone $(\mathrm{GH})$, prolactin (PRL), and proopiomelanocortin (POMC) mRNA during embryonic development. Quantitative real-time reverse transcription PCR was used to confirm microarray results for 31 genes.

Results: cDNA microarray identified 352 transcripts associated with increase in TSH- $\beta$ mRNA (based on cellular ontogeny) between embryonic days 10 and 17, and GH mRNA between embryonic days 12 and 17 while PRL mRNA did not increase until embryonic day 17. POMC mRNA levels also increased between embryonic days 10 and 17. There was a marked temporal pattern expression of 141 genes with that of TSH- $\beta$ mRNA. Similarly, the expression profiles of 69 and 61 genes indicate a potential involvement in the induction of GH and PRL mRNA, respectively. Quantitative real-time reverse transcription PCR confirmed microarray results for 31 genes.

Conclusion: A large number of candidate genes that could play a critical role in initiation of anterior pituitary hormone transcription in specialized cell types were identified. cDNA microarrays are capable of defining gene expression profiles during anterior pituitary cell differentiation. 
The development of the pituitary gland is similar in all vertebrates and has been studied extensively in the mouse. The anterior and intermediate lobes of the pituitary are derived from the oral ectoderm, while the posterior pituitary is derived from the neural ectoderm. Pituitary organogenesis and differentiation of specialized cells occurs in three major stages of differentiation. First, the pituitary placode is derived from thickening of the ectoderm at the roof of the primitive oral cavity that makes contact with the floor of the ventral diencephalon. Second, the oral ectoderm invaginates to form a rudimentary Rathke's pouch, while the ventral diencephalon evaginates to form the posterior pituitary. Third, a definitive Rathke's pouch is formed, and this is finally followed by the spatial and temporal differentiation of the various cell types within the mature anterior pituitary gland. A cascade of signaling molecules and transcription factors plays an important role in organ commitment, cell proliferation, cell patterning and terminal differentiation. Complex genetic interactions dictate normal development and the final product is the culmination of a coordinated process whereby repression and activation of target genes allows normal development to continue. This is the first study to evaluate changes in anterior pituitary gene expression during embryonic development of a species using microarrays technology. Numerous transcription factors and signaling molecules that had not previously been recognized as potentially implicated in pituitary development were identified. These findings represent a further step forward in the understanding of the complex signaling pathway and gene expression involved in pituitary cell differentiation. The functional significance of selected candidate genes through a definition of the effect of their overexpression on mRNA levels of pituitary hormones remain to be assessed.

References

1. Tsai J, Tong Q, Tan G, Chang AN, Orkin SH, Hotamisligil GS: The transcription factor GATA2 regulates differentiation of brown adipocytes. EMBO Rep 2005;6:879-884.

2. Machinis K, Pantel J, Netchine I, Leger J, Camand OJ, Sobrier ML, Dastot-Le Moal F, Duquesnoy P, Abitbol M, Czernichow P, Amselem S: Syndromic short stature in patients with a germline mutation in the LIM homeobox. Am J Hum Genet 2001;69:961-968.

3. Swerdlow AJ, Reddingius RE, Higgins CD, Spoudeas HA, Phipps K, Qiao Z, Ryder WD, Brada M, Hayward RD, Brook CG, Hindmarsh PC, Shalet SM: Growth hormone treatment of children with brain tumors and risk of tumor recurrence. J Clin Endocrinol Metab 2000;85:4444-4449.

4. Sklar CA, Mertens AC, Mitby P, Occhiogrosso G, Qin J, Heller G, Yasui Y, Robison LL: Risk of disease recurrence and second neoplasms in survivors of childhood cancer treated with growth hormone: a report from the Childhood Cancer Survivor Study. J Clin Endocrinol Metab 2002;87:3136-3141. 
